\title{
Optimization Letters Best Paper Award for 2018
}

\author{
Pavlo A. Krokhmal ${ }^{1}$ D . Oleg A. Prokopyev ${ }^{2}$ \\ Received: 4 November 2019 / Accepted: 12 December 2019 / Published online: 2 January 2020 \\ (c) Springer-Verlag GmbH Germany, part of Springer Nature 2020
}

\section{Dear Colleagues:}

It is our pleasure to announce that the following paper was named the winner of the 2018 OPTL Best Paper Award:

Frank E. Curtis, Zachary Lubberts, Daniel P. Robinson (2018), Concise complexity analyses for trust region methods, Optimization Letters 12(8), 1713-1724.

Abstract: Concise complexity analyses are presented for simple trust region algorithms for solving unconstrained optimization problems. In contrast to a traditional trust region algorithm, the algorithms considered in this paper require certain control over the choice of trust region radius after any successful iteration. The analyses highlight the essential algorithm components required to obtain certain complexity bounds. In addition, a new update strategy for the trust region radius is proposed that offers a second-order complexity bound.

Please join us in congratulating the authors of the paper, Dr. Curtis, Dr. Lubberts and Dr. Robinson, for their excellent contribution to literature!

The OPTL Best Paper Award carries a 1000 USD prize and a plaque. This year the Best Paper Award Selection Committee included OPTL Editorial Board members Dr. Sandra D. Eksioglu, Dr. Jean-Philippe Richard and Dr. Julius Žilinskas. We want to thank the members of the Committee for their service and Springer for sponsoring the award. We also would like to take this opportunity to invite nominations for the 2019 OPTL Best Paper Award. All papers published in OPTL during the year of 2019 are eligible.

Publisher's Note Springer Nature remains neutral with regard to jurisdictional claims in published maps and institutional affiliations.

Oleg A. Prokopyev

droleg@pitt.edu

1 University of Arizona, Tucson, USA

2 University of Pittsburgh, Pittsburgh, USA 